\title{
Literature Review on Korean Medicine Treatment for Alopecia
}

\author{
Seul Woo Leem ${ }^{1 \dagger}$, Min Kyeong Kim ${ }^{1 \dagger}$, Seo Lim Ko${ }^{1}$, Hye In Jeong ${ }^{2}$, Kyeong Han Kim ${ }^{3,4}$ * \\ ${ }^{1}$ Korean Mecince, College of Korean Medicine, Woosuk University, Wanju, Republic of Korea \\ ${ }^{2}$ Department of Preventive Medicine, College of Korean Medicine, Kyung Hee University, Seoul, Republic of Korea \\ ${ }^{3}$ Woosuk Institute of Smart Convergence Life Care (WSCLC), Woosuk University, Wanju, Republic of Korea \\ ${ }^{4}$ Department of Preventive Medicine, College of Korean Medicine, Woosuk University, Wanju, Republic of Korea
}

Received July 7, 2021

Reviewed July 22, 2021

Accepted August 23, 2021

\section{*Corresponding Author}

Kyeong Han Kim

Department of Preventive Medicine,

College of Korean Medicine, Woosuk University, 443, Samnye-ro, Samnye-eup, Wanju 55338, Republic of Korea Tel: +82-63-290-9031

E-mail: solip922@hanmail.net

${ }^{\dagger} S W L$ and MKK contributed equally to this work.
This study aimed to analyze the use of Korean medicine treatments for alopecia in among clinical studies. We identified and analyzed 22 studies from Korean databases; Oriental Medicine Advanced Searching Integrated System (OASIS), Science ON, Korean Studies Information Service System (KISS), and Research Information Sharing Service (RISS) and international database; PubMed. We analyzed the Korean medical treatment in each case and determined the tendency to use each intervention. We analyzed 1,464 patients from 22 selected studies. Herbal medicine, acupuncture, external medicine or products, pharmacopuncture, and phototherapy were used for alopecia treatment. The herbal medicines mainly used to treat alopecia were Gagam Cheongyoung-tang, Gagam Hwajung-hwan, and Yukmijihwang-tang-hwan. The acupoints primarily used were GV20, EX-HN1, GB5, KI3, PC6, ST36, GV22, and A-shi. The most commonly used pharmacopuncture therapies were Hwangryunhaedoktang (HH), Carthami Fructus (CF), Bee Venom (BV), and Hominis placenta (HP). The Korean medical treatment for alopecia improved the condition of patients. However, seven studies reported the occurrence of side effects such as pruritus, dazed, drowsiness, headache, pain, and diarrhea. This study shows the potential of Korean medicine for the treatment of alopecia. Further studies with a large sample size and long-term follow-up are warranted to establish the primary treatment guidelines and objective outcome measures for alopecia.

Keywords: alopecia, hair loss, korean medicine, alopecia areata, androgenetic alopecia

\section{INTRODUCTION}

Alopecia, a disease that results in hair loss in areas where hair normally exists [1], is associated with a negative change in the appearance of the scalp due to gradual decrease in hair density [2]. In Korea, the number of patients with alopecia has increased in the last 5 years (from 208,534 in 2016 to 210,408 in 2020) [3]. In addition, alopecia has been increasingly affected by not only aging or genetic factors but also acquired factors such as environmental pollution, stress, and hormonal secretion abnormalities caused by dietary changes [4].

Alopecia can be divided into cicatricial alopecia and noncicatricial alopecia. Cicatricial alopecia refers to permanent hair loss, whereas non-cicatricial alopecia refers to recoverable hair loss. Non-cicatricial alopecia includes alopecia areata, androgenetic alopecia, and telogen effluvium [5].

Androgenetic alopecia is the most common type of hair loss in both men and women. Androgenic and genetic predispositions appear to be important factors in androgenetic alopecia [6]. Alopecia areata is the formation of round or oval patches of alopecia of various sizes. It is divided into patchy alopecia areata (partial loss of scalp hair), alopecia totalis (total loss of scalp hair), and alopecia universalis (total loss of body and scalp hair) [7].

Currently, the most common treatments for alopecia are oral Finasteride, a Food and Drug Administration-approved drug, 
and topical Minoxidil [8]. However, Finasteride treatment takes 6-12 months to reduce hair loss or increase hair growth and recurrence of alopecia is observed within 2 months of discontinuation. In addition, the use of Finasteride is restricted because of side effects such as reduced sexual desire, decreased sexual functions such as erectile dysfunction, and the risk of birth defects in women. Further, the use of Minoxidil has limitations such as it should be applied twice a day consistently and it works only if the medication stays on the scalp for 3-4 hours [9]. In particular, the use of Minoxidil can cause side effects such as dizziness, dry scalp, itching, skin irritation, and erythema [10]. Treatments such as hair transplant have been proposed; however, these treatments have side effects such as skin atrophy, blood vessel enlargement, hormonal imbalance, and growth inhibition because of long-term drug application [11].

The use of Western medical treatments and hair transplantation has limitations such as side effects; hence, it is important to develop a safe and effective treatment method in Korean medicine.

In Korean medicine, alopecia is generally diagnosed into four syndrome-blood heat syndrome, qi stagnation and blood stasis syndrome, qi and blood deficiency syndrome, and liver and kidney deficiency syndrome. Based on the DeficiencyExcess syndrome classification, deficiency syndromes are classified into blood deficiency syndrome, qi and blood deficiency syndrome, and liver and kidney deficiency syndrome, and excess syndromes are classified into blood heat syndrome, windheat syndrome, qi stagnation, and blood stasis syndrome [12, 13]. The various treatment methods used for alopecia include herbal medicine [14], which involve diagnosis and treatment based on an overall analysis of symptoms and signs, and external treatments such as acupuncture [15], pharmacopuncture [16], and phototherapy [17]. Recently, various case reports and experimental studies have reported the use of microneedling [19] and needle embedding [20] for the treatment of alopecia.

Various Korean medical treatments have been developed and used owing to the growing demand for hair loss treatment; however, the consistency and direction of treatment methods have not been established sufficiently. We conducted this study to establish future treatment directions and effective treatments for alopecia by analyzing case reports and clinical studies on Korean medical treatments for alopecia reported in Korean database and PubMed, a foreign database.

\section{MATERIALS AND METHODS}

\section{Data sources}

May 7, 2021, the study was conducted using four Korean databases-Oriental Medicine Advanced Searching Integrated System (OASIS), Science ON, Korean Studies Information Service System (KISS), and Research Information Sharing Service (RISS) - and one international database-PubMed.

We used "Alopecia" as the search term in OASIS and "(Alopecia) AND (Herbal medicine OR Pharmacopuncture OR Electroacupuncture OR Acupuncture OR Moxibustion OR Cupping Therapy)" in Science ON, KISS, and RISS to search for papers on Korean medical treatment for improving alopecia. We searched PubMed using the following search strategy: ("Acupuncture" [MeSH] OR "Electroacupuncture" [MeSH] OR "Cupping Therapy" [MeSH] OR "Dry Needling" [MeSH] OR "Moxibustion" [MeSH] OR "Plants, Medicinal" [MeSH] OR "Phytotherapy" [MeSH] OR "Pharmacognosy" [MeSH] OR "Ethnobotany" [MeSH] OR "Ethnopharmacology" [MeSH]) AND ("Alopecia" [MeSH]).

\section{Inclusion and exclusion criteria of studies}

In this study, we reviewed papers published in Korean and international journals on Korean medicine treatments to improve alopecia. This study analyzed the use of several treatments methods such as herbal medicine, acupuncture, electropuncture, pharmacopuncture, moxibustion, and cupping therapy.

We excluded non-human experimental studies, reviews, protocol studies, concise reports, and studies unrelated to alopecia treatment. In addition, we excluded studies with interventions unrelated to the direction of the study, studies without original text, and studies with symptoms or diseases unrelated to alopecia.

\section{Data extraction}

In total, 22 articles were included. The following data were collected: publication year, study type, number of cases. In addition, we analyzed the type of alopecia, Korean medical treatment used, outcome measurements, treatment results, and side effects. 


\section{RESULTS}

\section{Search results}

We found 144 articles using Korean databases and 77 articles using the international database. A total of 174 articles were obtained after all articles were ordered in Excel and duplicates based on author names, publication date, and title were removed. Titles and abstracts of 174 articles were reviewed, and 29 non-human experimental studies, 36 reviews, one concise report, one protocol study, and 56 articles unrelated to alopecia treatment were excluded. Finally, we reviewed the full text of the remaining 51 articles. A total of 22 articles were included in analysis after excluding two articles that were not found, 21 articles unrelated to intervention, and six articles on alopecia with other diseases (Fig. 1, Table 1, 2).

\section{Number of patients}

A total of 1,464 patients received alopecia treatment in 22 studies on Korean medical treatments for alopecia. Among the 1,464 patients, 980 were men and 484 were women.
Potentially relevant studies identified in electronic Korean databases $(n=144)$
Potentially relevant studies identified in electronic English databases $(n=77)$

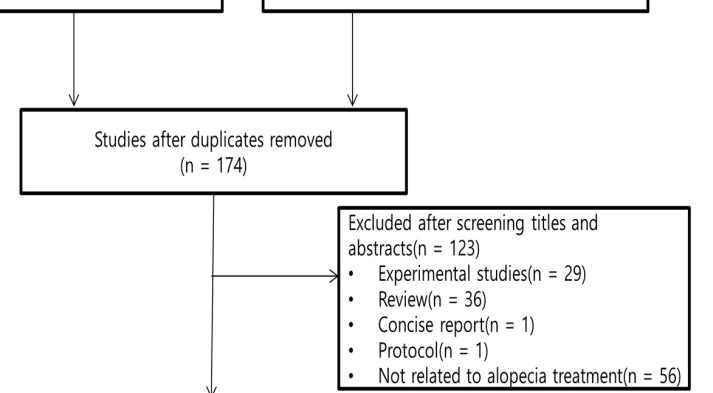

Full-text studies assessed for eligibility $(n=51)$

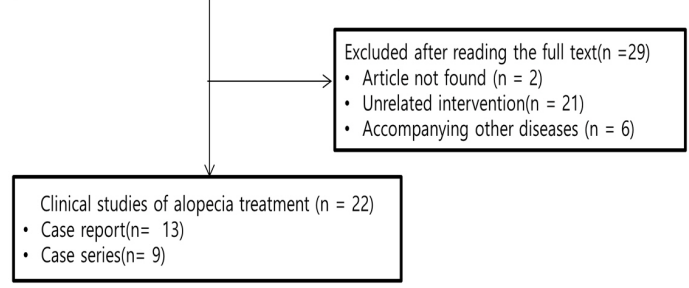

Figure 1. Flow chart.

\section{Classification by alopecia types}

Six types of alopecia were found in the selected 22 studies. The frequency of each type of alopecia was as follows: alopecia areata, 1,286; androgenetic alopecia, 139; febrile alopecia, 30; telogen effluvium, 7; and alopecia totalis and alopecia universalis, 1 (Table 3).

\section{Classification by treatments}

The treatments used in 22 studies were herbal medicine, acupuncture, pharmacopuncture, external medicine or product, phototherapy, cupping therapy, life management, microneedling, needle-embedding, blood-letting therapy, moxibustion (Zhuang medicine) and galvanic therapy. The frequency of use of each treatment was as follows (papers): herbal medicine, 17; acupuncture, 15; pharmacopuncture, 9; external medicine or product, 7; phototherapy, 4; cupping therapy, life management, microneedling, and needle embedding, 2; and blood-letting therapy, moxibustion (Zhuang medicine), and galvanic therapy, 1 (Table 4).

In subsection, small-scale case reports $(<$ six) accounted for all of those cases, and large-scale case reports (>six) were calculated by treating them as one. In addition, if different types of treatments were used in one case, each treatment was considered to have been used once.

\section{Herbal medicine}

In the case of combined prescriptions such as Samul-tang plus Yukmijihwang-tang, each prescription was considered to have been used once. Gamibang and Gagambang were considered to be of the same type as the original prescription. For example, Hyeongbangjihwang-tang gamibang was considered to be the same type as Hyeongbangjihwang-tang. Hwan was treated as Tang.

Herbal medicine was used in 25 of 30 cases. The names of the prescribed herbal medicines were available in 23 cases. A total of 18 types of herbal medicines were prescribed. In the order of frequency, Gagam Cheongyoung-tang and Gagam Hwajung-hwan were prescribed six times; Yukmijihwang-tang-hwan was prescribed three times; and Samul-tang, Soshiho-tang, and Hyungbangjihwang-tang gamibang were prescribed two times. In addition to Yangdokbeakho-tang gamibang, Shihogayonggolmoryeo-tang, Baekhogainsam-tang, Galgeun-tang, Banha- 


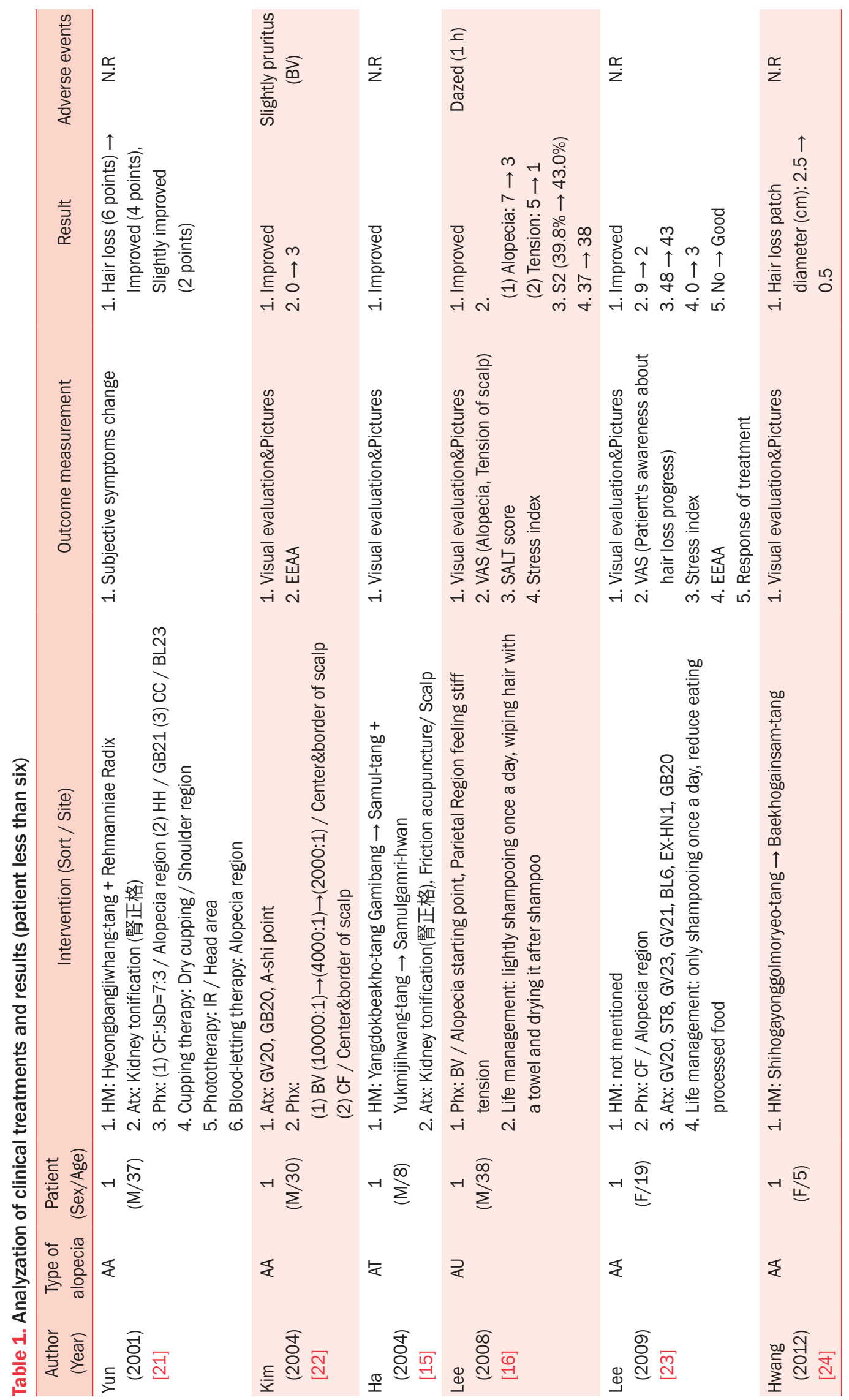




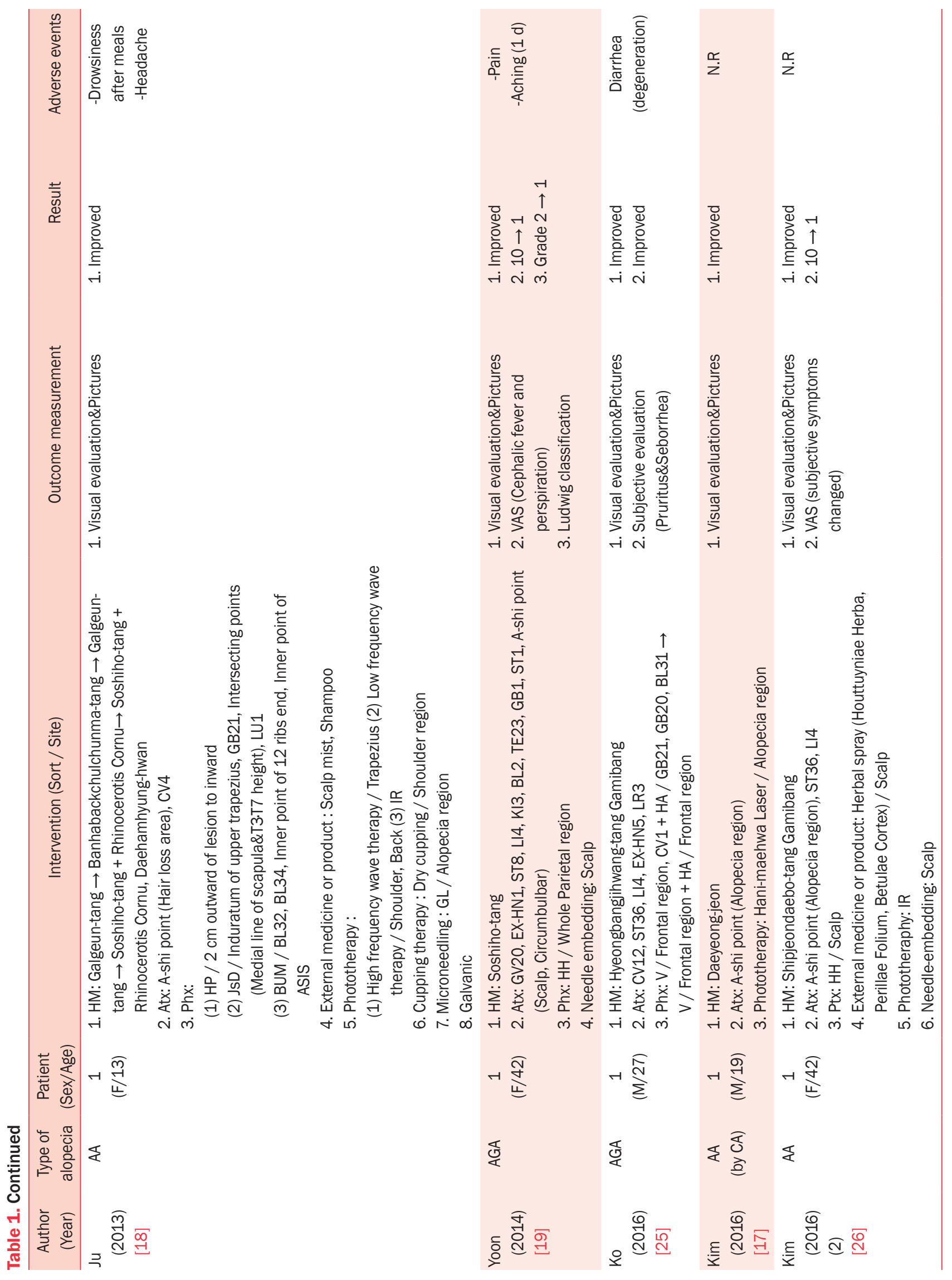




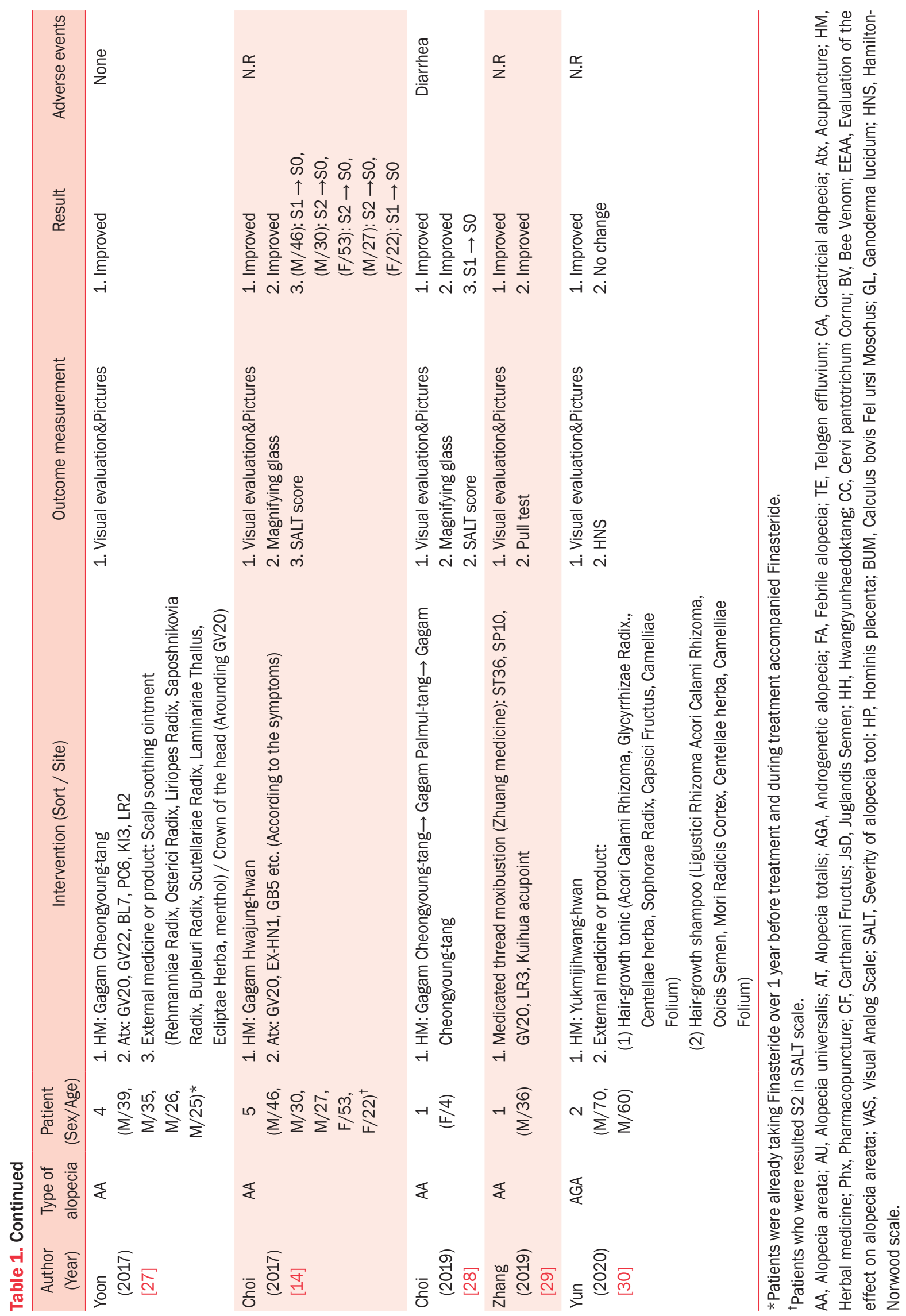




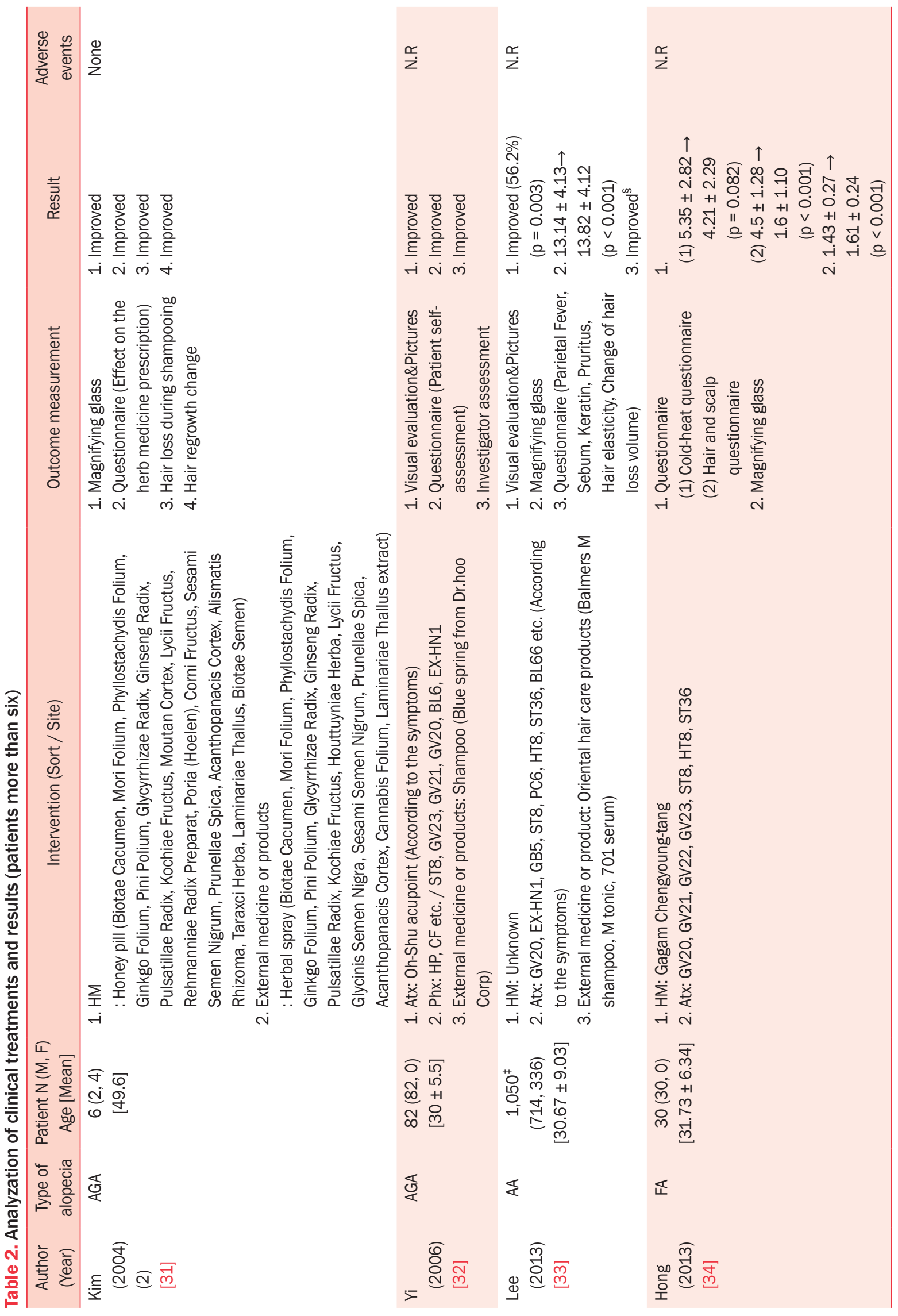




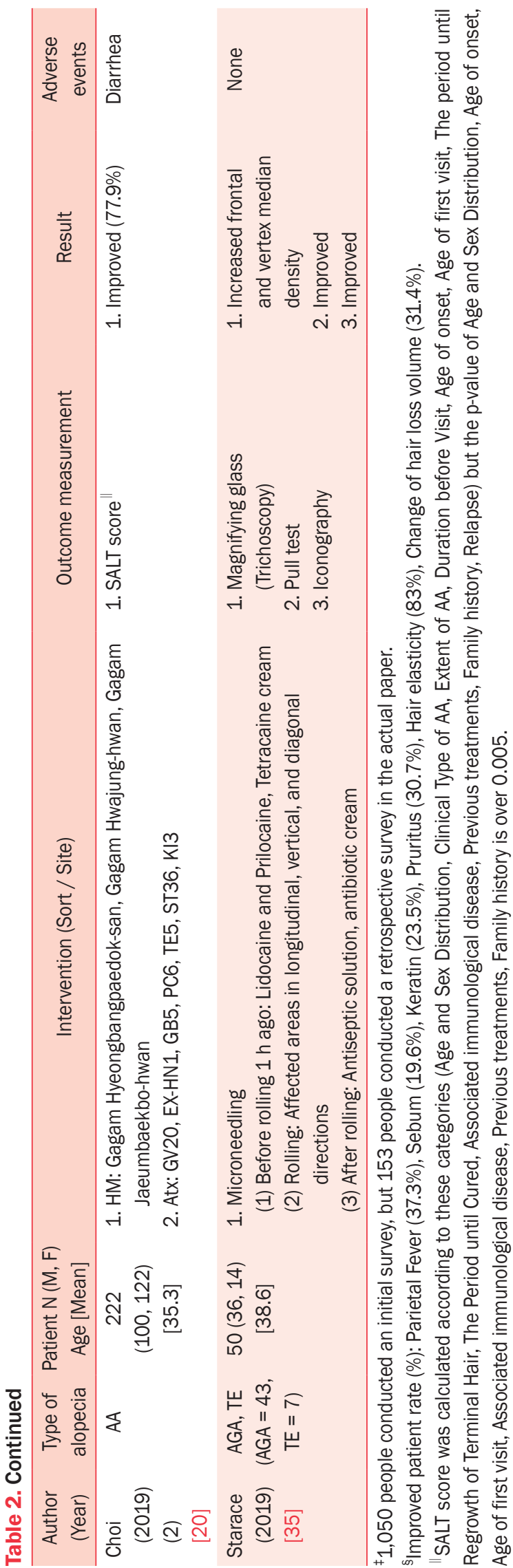

Table 3. Types of alopecia reported in alopecia clinical studies

\begin{tabular}{lc}
\hline \multicolumn{1}{c}{ Type of alopecia } & Number of patients n (\%) \\
\hline Alopecia areata & $1,286(87.8 \%)$ \\
Androgenetic alopecia & $139(9.5 \%)$ \\
Febrile alopecia & $30(2.0 \%)$ \\
Telogen effluvium & $7(0.5 \%)$ \\
Alopecia totalis, Alopecia universalis & $1(0.1 \%)$ \\
Total & 1,464 \\
\hline
\end{tabular}

Table 4. Interventions reported in alopecia clinical studies

\begin{tabular}{|c|c|c|}
\hline Intervention & $\begin{array}{c}\text { Number of papers } \\
n(\%)\end{array}$ & $\begin{array}{c}\text { Number of cases } \\
n(\%)\end{array}$ \\
\hline Herbal medicine & $17(77.3 \%)$ & $25(83.3 \%)$ \\
\hline Acupuncture & $15(68.2 \%)$ & $22(73.3 \%)$ \\
\hline Pharmacopuncture & $9(40.9 \%)$ & $9(30.0 \%)$ \\
\hline External medicine or product & 7 (31.8\%) & $11(36.7 \%)$ \\
\hline Phototherapy & $4(18.2 \%)$ & $4(13.3 \%)$ \\
\hline $\begin{array}{l}\text { Cupping therapy, Life } \\
\text { management, Microneedling, } \\
\text { Needle-embedding }\end{array}$ & $2(9.1 \%)$ & $2(6.7 \%)$ \\
\hline $\begin{array}{l}\text { Blood-letting therapy, } \\
\text { Moxibustion (Zhuang } \\
\text { medicine), Galvanic }\end{array}$ & $1(4.5 \%)$ & $1(3.3 \%)$ \\
\hline Total & 22 & 30 \\
\hline
\end{tabular}

Table 5. Herbal medicines reported in alopecia clinical studies

\begin{tabular}{|c|c|}
\hline Herbal medicine & $\begin{array}{l}\text { Number of } \\
\text { cases } n(\%)\end{array}$ \\
\hline $\begin{array}{c}\text { Gagam-cheongyoung-tang (加減淸營湯), } \\
\text { Gagam-hwajung-hwan (加減和中丸) }\end{array}$ & $6(18.2 \%)$ \\
\hline Yukmijihwang-tang · huan (六味地黃湯· 丸) & $3(9.1 \%)$ \\
\hline $\begin{array}{l}\text { Samul-tang (四物湯) (including Gami) } \\
\text { Soshiho-tang (小柴胡湯) (including Gami) } \\
\text { Hyeongbangjihwang-tang gamibang (荊防地黃湯加味方) }\end{array}$ & $2(6.1 \%)$ \\
\hline $\begin{array}{l}\text { Yangdokbeakho-tang (陽毒白虎湯) } \\
\text { Shihogayonggolmoryeo-tang (柴胡加龍骨牡蠣湯) } \\
\text { Baekhogainsam-tang (白虎加人參湯) } \\
\text { Galgeun-tang (葛根湯) } \\
\text { Banhabackchulchunma-tang (半夏白术天麻湯) } \\
\text { Daehamhyung-hwan (大陷胸丸) } \\
\text { Daeyeong-jeon (大營煎) } \\
\text { Gagam Palmul-tang (加減八物湯) } \\
\text { Gagam Hyeongbangpaedok-san (加減荊防敗毒散) } \\
\text { Gagam Jaeumbaekbo-hwan (加減滋陰百補丸) } \\
\text { Samulgamri-hwan (四物坎离丸) } \\
\text { Honey pill (蜜丸) }\end{array}$ & $1(3.0 \%)$ \\
\hline Total & 33 \\
\hline
\end{tabular}


backchulchunma-tang, Daehamhyung-hwan, Daeyeong-jeon, Gagam Palmul-tang, Gagam Hyeongbangpaedok-san, Gagam Jaeumbaekbo-hwan, and Samulgamri-hwan, Honey pills were used once (Table 5).

\section{Acupuncture}

Acupuncture was used in 22 of the 30 cases analyzed. In order of frequency, the following acupuncture points were used: GV20, 15 times; EX-HN1, nine times; GB5, seven times; KI3 and PC6, six times; ST36, GV22, and A-shi point, five times; BL7, LR2, and ST8, four times; LI4, three times; and Kidney tonification, GV23, GV21, GB20, and HT8, two times. In addition, BL6, CV4, BL2, TE23, GB1, ST1, CV12, EX-HN5, LR3, BL66, TE5 and Oh-shu acupoint were used once (Table 6).

\section{Pharmacopuncture}

Pharmacopuncture treatment was used in nine of the 30 cases analyzed. Hwangryunhaedoktang $(\mathrm{HH})$ and Carthami Fructus (CF) pharmacopuncture were the most commonly

Table 6. Acupoints reported in alopecia clinical studies

\begin{tabular}{lc}
\hline \multicolumn{1}{c}{ Acupoint } & $\begin{array}{c}\text { Number of cases } \\
\mathrm{n}(\%)\end{array}$ \\
\hline GV20 & $15(15.8 \%)$ \\
EX-HN1 & $9(9.5 \%)$ \\
GB5 & $7(7.4 \%)$ \\
KI3, PC6 & $6(6.3 \%)$ \\
ST36, GV22, A-shi point & $5(5.3 \%)$ \\
BL7, LR2, ST8 & $4(4.2 \%)$ \\
\hline LI4 & $3(3.2 \%)$ \\
Kidney tonification, GV23, GV21, GB20, HT8 & $2(2.1 \%)$ \\
BL6, CV4, BL2, TE23, GB1, ST1, CV12, EX-HN5, & $1(1.1 \%)$ \\
$\quad$ LR3, BL66, TE5, Oh-Shu acupoint & \\
\hline Total & 95 \\
\hline
\end{tabular}

Table 7. Pharmacopunctures reported in alopecia clinical studies

\begin{tabular}{lc}
\hline \multicolumn{1}{c}{ Pharmacopuncture } & $\begin{array}{c}\text { Number of cases } \\
\mathrm{n}(\%)\end{array}$ \\
\hline $\mathrm{HH}, \mathrm{CF}$ & $3(18.8 \%)$ \\
$\mathrm{BV}, \mathrm{HP}$ & $2(12.5 \%)$ \\
$\mathrm{JSD}, \mathrm{CC}, \mathrm{BUM}, \mathrm{V}, \mathrm{HA}, \mathrm{CF} \cdot \mathrm{JSD}$ (Mixed) & $1(6.3 \%)$ \\
Total & 16 \\
\hline
\end{tabular}

used pharmacopuncture treatments (three times), followed by Bee Venom (BV) and Hominis placenta (HP) (two times), and Juglandis Semen (JsD), Cervi pantotrichum Cornu , Calculus bovis Fel ursi Moschus, V, HA and CF plus JsD (one time) (Table 7).

$\mathrm{V}$ pharmacopuncture consists of the cystic stone of Bos taurus domesticus Gmelin, the gallbladder of Ursus thibetanus G. Cuvier, the gland secretion of Moschus moschiferus L, etc. HA pharmacopuncture consists of the flower of Lonicera japonica Thunb, the fruit of Eriobotrya japonica Lindl, etc.

\section{External medicine or product}

External medicine or product was used in 11 of the 30 cases analyzed. The most commonly used external medicine or product was shampoo (five times), followed by ointment (four times), tonic (three times), herbal spray (two times), and serum and mist (one time) (Table 8).

\section{Phototherapy}

Phototherapy was used in four of the 30 cases analyzed. Infrared radiation was the most commonly used phototherapy. High-frequency, low-frequency and laser were used once each. Hani-maehwa Laser, a low-power laser, was used (Table 9).

Table 8. External medicine or products reported in clinical studies

\begin{tabular}{lc}
\hline External medicine or product & $\begin{array}{c}\text { Number of papers } \\
\mathrm{n}(\%)\end{array}$ \\
\hline Shampoo & $5(31.3 \%)$ \\
Ointment & $4(25.0 \%)$ \\
\hline Tonic & $3(18.8 \%)$ \\
Herbal spray & $2(12.5 \%)$ \\
Serum, Mist & $1(6.3 \%)$ \\
Total & 16 \\
\hline
\end{tabular}

Table 9. Phototherapies reported in clinical studies

\begin{tabular}{lc}
\hline \multicolumn{1}{c}{ Phototherapy } & $\begin{array}{c}\text { Number of cases } \\
\mathrm{n}(\%)\end{array}$ \\
\hline IR (Infrared ray) & $3(50.0 \%)$ \\
\hline High frequency, Low frequency, Laser & $1(16.7 \%)$ \\
\hline Total & 6 \\
\hline
\end{tabular}




\section{Other interventions}

The other types of treatments used were cupping therapy, life management, microneedling, needle embedding, blood-letting therapy, moxibustion (Zhuang medicine) and galvanic therapy. Cupping therapy, life management, microneedling, and needle embedding were used twice, and blood-letting therapy, moxibustion (Zhuang medicine) and galvanic therapy were used once (Table 10).

\section{Classification by outcome measurement}

The treatment can change for each case in the same study; however, the outcome measurements are always the same. Therefore, we assessed the outcome by the number of papers and not by the number of cases. In total, 18 types of outcome measurements were used in 22 alopecia treatment studies. Eight types of evaluation tools were used in two or more papers. visual observation and images, 16 studies; magnifying glass, 6 studies; Visual Analog Scale (VAS), Severity of alopecia tool (SALT), and questionnaire, 4 studies; and stress index, Evaluation of the effect on alopecia areata (EEAA), and pull test, 2 studies. In ad-

Table 10. Other interventions reported in clinical studies

\begin{tabular}{lc}
\hline \multicolumn{1}{c}{ Other intervention } & $\begin{array}{c}\text { Number of cases } \\
\mathrm{n}(\%)\end{array}$ \\
\hline $\begin{array}{l}\text { Cupping therapy, Life management, Microneedling, } \\
\text { Needle embedding }\end{array}$ & $2(18.2 \%)$ \\
$\begin{array}{l}\text { Blood-letting therapy, Moxibustion (Zhuang } \\
\text { medicine), Galvanic }\end{array}$ & $1(9.1 \%)$ \\
Total & 11 \\
\hline
\end{tabular}

Table 11. Outcome measurements reported in clinical studies

\begin{tabular}{lc}
\multicolumn{1}{c}{ Outcome measurement } & $\begin{array}{c}\text { Number of papers } \\
\mathrm{n}(\%)\end{array}$ \\
\hline Visual evaluation\&Pictures & $16(72.7 \%)$ \\
Magnifying glass & $6(27.3 \%)$ \\
VAS, SALT, Questionnaire & $4(18.2 \%)$ \\
Stress index, EEAA, Pull test & $2(9.1 \%)$ \\
Subjective symptoms change, subjective & $1(4.5 \%)$ \\
evaluation, response of treatment, Ludwig & \\
Classification, HNS, hair condition change, hair & \\
loss during shampooing, hair regrowth change, & \\
investigator assessment, iconography & 22 \\
\hline Total & \\
\hline
\end{tabular}

dition to the following outcome measurements were used once; subjective symptoms change, subjective evaluation, response to treatment, Ludwig classification, Hamilton-Norwood scale (HNS), hair condition change, hair loss during shampooing, hair regrowth change, investigator assessment, and iconography (Table 11).

The aforementioned outcome measurements can be largely divided into visual, subjective, and objective measurements. Visual measurements (visual evaluation and pictures, magnifying glass, severity on the alopecia tool, evaluation of the effect on alopecia areata, Ludwig classification, Hamilton-Norwood scale, hair condition change, and iconography) were used 32 times. Subjective measurements (VAS, questionnaire, stress index, subjective symptoms change, subjective evaluation, response of treatment, and investigator assessment) were used 14 times. Objective measurements (pull test, hair loss during shampooing, and hair regrowth change) were used four times (Table 12).

\section{Side effects}

In 12 studies, side effects were not mentioned. Three studies reported no side effects. In the remaining seven studies, the side effects were described. Choi (2019) [20] and Choi (2019) (2) [28] reported diarrhea, Ko (2016) [25] reported worsening of diarrhea symptoms in patients with diarrhea, Kim (2004) [22] reported slight pruritus during treatment with BV, Lee (2008) [16] reported dizziness for an hour, Ju (2013) [18] reported drowsi-

Table 12. Outcome measurements reported in clinical studies (divided into 3 groups)

\begin{tabular}{|c|c|c|}
\hline Type & Outcome measurement & $\begin{array}{c}\text { Number of } \\
\text { total outcome } \\
\text { measurement } \\
n(\%)\end{array}$ \\
\hline Visual & $\begin{array}{l}\text { Visual evaluation\&Pictures, } \\
\text { Magnifying glass, SALT, EEAA, Ludwig } \\
\text { Classification, HNS, hair condition } \\
\text { change, iconography }\end{array}$ & $32(64 \%)$ \\
\hline Subjective & $\begin{array}{l}\text { VAS, Questionnaire, Stress index, } \\
\text { subjective symptoms change, } \\
\text { subjective evaluation, response of } \\
\text { treatment, investigator assessment }\end{array}$ & $14(28 \%)$ \\
\hline Objective & $\begin{array}{l}\text { Pull test, hair loss during shampooing, } \\
\text { hair regrowth change }\end{array}$ & $4(8 \%)$ \\
\hline Total & & 50 \\
\hline
\end{tabular}


ness after meals and headache, and Yoon (2014) [19] reported pain and aching for a day.

\section{DISCUSSION}

Recently, the incidence of alopecia, a hair loss disease, has been increasing $[1,3]$. Currently, Finasteride and Minoxidil are mainly used in Western medicine for the treatment of alopecia [8]. However, they can cause problems such as decrease sexual function and increase the risk of birth defects [9]. Hence, we investigated treatments for alopecia in Korean medicine, which has relatively few side effects.

A total of 22 studies were selected for analysis. These studies have been published in the past 20 years (one in 2001, three in 2004, 1 in 2006, one in 2008, one in 2009, one in 2012, three in 2013, one in 2014, three in 2016, two in 2017, four in 2019, and one in 2020). This suggests that studies on treatment for alopecia in Korean medicine are steadily underway.

In 22 studies, 1,464 patients (980 men and 484 women) were treated for alopecia using Korean medical treatments. A total of six types of alopecia-alopecia areata, androgenetic alopecia, febrile alopecia, telogen effluvium, alopecia totalis, and alopecia universalis-were identified in 1,464 patients. Most patients $(\mathrm{n}=1,286)$ had alopecia areata, while 139 patients had androgenetic alopecia. This gap is believed to have been particularly large because a case study [33] included 1050 patients with alopecia areata.

The treatments for alopecia in Korean medicine include herbal medicine, acupuncture, pharmacopuncture, external medicine or product, cupping therapy, microneedling, moxibustion (Zhuang medicine) and blood-letting therapy. In addition, phototherapy, life management, and galvanic therapy are used as a supplement therapy. However, Yoon (2017) [27] used Finasteride along with Korean medication to treat alopecia. To determine the frequency of each Korean medical treatment, small-scale case reports ( $<6$ cases) were considered each, and large-scale case reports ( $>6$ cases) were calculated by treating the number of cases as one.

Herbal medicine was used in 25 of 30 cases. The most commonly used prescriptions were Gagam Cheongyoung-tang and Gagam Hwajung-hwan, six times each. Cheongyoung-tang, a typical prescription for clearing heat and cooling blood, nourishes the liver and kidney to cool the heat. Gagam Cheongyoung-tang is a basic addition to Cheongyoung-tang [36]. As mentioned in the introduction, the cause of alopecia is blood- heat syndrome, wind-heat syndrome, and dampness-heat syndrome [12], thus, the use of Gagam Cheongyoung-tang which has the effect of clearing heat can control alopecia. Gagam Hwajung-hwan is used in patients with stress heat syndrome accompanied by digestive congestion. Currently, alopecia is considered to be affected by acquired factors such as stress [23]; therefore, it is believed that alopecia can be controlled by Gagam Hwajung-hwan.

Acupuncture was used in 22 of 30 cases. The most commonly used acupoint was GV20, which was used 15 times. This acupoint is located on the scalp and is believed to have been used to relieve scalp fever, a major cause of alopecia. EX-HN1, GB5, GV22, and A-shi point are all local acupuncture points used more than five times. Local acupuncture is considered to induce new hair by increasing hair cells, dermal papilla cells, and capillary blood flow circulation, which play an important role in hair development [21]. The distal acupuncture points were KI3, PC6, and ST36, which were used more than five times. ST36 is usually used for alopecia caused by qi and blood deficiency syndrome [26].

Pharmacopuncture was used in 9 of 30 cases. The most commonly used pharmacopuncture was $\mathrm{HH}$ and $\mathrm{CF}$. HH pharmacopuncture was used to control the heat in the parietal region by clearing away heat toxin and improve alopecia by stimulating the scalp [37]. CF pharmacopuncture is effective in activating blood circulation and detoxification; hence, it is likely to be effective in a syndrome in which the hair coat cannot be nourished due to body fluid deficiency, blood deficiency, and poor communication between blood circulation [23]. In addition, BV and HP pharmacopuncture were used. Ko (2016) [25] reported the use of HN pharmacopuncture; however, on analyzing the details, $\mathrm{HA}$ acupuncture, not $\mathrm{HN}$ acupuncture, was reported. Hence, we determined and analyzed it as HA acupuncture.

External medicine or product was used in 11 of 30 cases. The most commonly used external medicine or product was shampoo (five times), which is thought to be versatile because it is closely involved in scalp health and alopecia. Ointment was the second most commonly used product to soothe the scalp, rather than treat alopecia directly. Yoon (2017) [27] reported the use of a self-made sedation ointment of the root of Rhemannia glutinosa (Gaertner) Liboschitz, the root of Ostericum koreanum Kitagawa, and the tuberous root of Liriope platyphylla Wang et Tang to be applied on the head.

Phototherapy was used in 4 of 30 cases. The most commonly used phototherapy was infrared radiation, which is believed 
to have been used to aid the supply of nutrients to hair by promoting blood circulation and repair the scalp damaged by cell activation [38]. A high frequency, low frequency, and laser were used once each. High-frequency laser was used to administer heat to the deep parts of the body, and low-frequency laser was used for systemic stimulation of nerves and muscles [18]. The Hani-maehwa laser was used to induce hair growth and reduce the side effects [17].

The other treatments included cupping therapy, microneedling, needle embedding, moxibustion (Zhuang medicine), blood-letting therapy, life management, and galvanic therapy. Regarding cupping, dry cupping was performed in the shoulder region. Microneedling facilitates the absorption of pharmacopuncture solution into the skin and treatment of alopecia through scalp irritation [18]. Needle-embedding therapy was performed using a $29 \mathrm{Gx} 30 \mathrm{~mm}$ Miracu in the parietal region of the scalp. This treatment induces the body's defense system by introducing harmless foreign substances into the bloodstream, which is believed to facilitate alopecia treatment by stimulating the parietal region [26].

Regarding life management, Lee (2008) [16] indicated that hair should be shampooed lightly once a day, wiped lightly with a towel, and dried with a dryer. Lee (2009) [23] set a standard for shampooing once a day and reducing the consumption of processed foods. Yun (2001) [21] indicated that blood circulation should be increased in the alopecia area through bloodletting therapy, and Ju (2013) [18] reported scalp care before acupuncture treatment with galvanic.

In 22 studies, 18 types of outcome measurements (50 times) were used. Outcome measurements were largely divided into visual, subjective, and objective measurements, Visual measurements were used the most frequently (32/50 [64\%]), with a high proportion of visual evaluations and pictures. This is because alopecia is a visible disease, and visual evaluation methods are simple and accurate. SALT, one of the popular hair loss assessment tools, consists of visual indicators. This is related to the use of more subjective tools than objective evaluation tools.

This study has limitations. To assess the trend of foreign research, we used PubMed; however, the PubMed search yielded only two studies that were in accordance with the eligibility criteria. In addition, the assessment of objective outcome measurements for alopecia is essential in the future, given that most of the outcome measurements used in the studies were far from objective, forcing the authors to intervene in subjective interpretation. Most selected studies were case studies; hence, in many studies, interventions changed as the treatment progressed. For example, in Ju's study (2013) [18], the herbal medicine administered to a patient was changed to Galgeun-tang, Banhabaekchulcheonma-tang, and Soshiho-tang plus the horn of Cervus elaphus L., Daehamhyung-hwan four times. Thus, further studies are warranted to determine the therapeutic effectiveness of each intervention.

Compared with a previous review in 2019 [39], we analyzed six more studies, including those from foreign databases. Moreover, we corrected the incorrect data in the previous review. We hope that this study will be used as a reference to develop guidelines for alopecia for actual clinical use.

\section{CONCLUSION}

This study analyzed 22 studies on Korean medicine treatments for alopecia in using four Korean databases and a international database. The main findings of this study are as follows:

1. A total of 1,464 patients were included in 22 studies. Patients with alopecia areata accounted for $87.8 \%$ of all patients. The treatments used for alopecia, in order of frequency (cases), were as follows: herbal medicine, acupuncture, external medicine or product, pharmacopuncture, phototherapy, cupping therapy, life management, microneedling, needle embedding, blood letting therapy, moxibustion (Zhuang medicine) and galvanic therapy.

2. In herbal medicine, Gagam Cheongyoung-tang and Gagam Hwajung-hwan, Yukmijihwang-tang-hwan, Samul-tang, Soshiho-tang, and Hyungbangjihwang-tang gamibang were mainly used.

3. The most commonly used acupuncture point was GV20, followed by EX-HN1, GB5, KI3, PC6, ST36, GV22, and A-shi. In addition, both local and distal acupuncture treatments were performed; however, the proportion of local acupuncture treatments was higher, with the top eight most commonly used acupoints. $\mathrm{HH}$ and $\mathrm{CF}$ were mainly used in pharmacopuncture.

4. The main external medicine or product used was shampoo, followed by ointment. The phototherapy mainly used was infrared radiation, followed by high-frequency, low-frequency and laser.

5. The outcome measurements were largely divided into visual, subjective, and objective measurements. Visual measurements (such as visual evaluation and pictures and magnifying glass) were the most commonly used. The other outcome mea- 
sures used were VAS, SALT, questionnaire, and stress index.

\section{CONFLICTS OF INTEREST}

The authors declare no conflict of interest.

\section{AUTHORS' CONTRIBUTION}

SWL and MKK contributed equally to this work

\section{FUNDING}

This paper was supported by Woosuk University.

\section{ORCID}

Seul Woo Leem, https://orcid.org/0000-0001-9521-2126

Min Kyeong Kim, https://orcid.org/0000-0002-7725-352X

Seo Lim Ko, https://orcid.org/0000-0003-3288-8094

Hye In Jeong, https://orcid.org/0000-0002-3651-9678

Kyeong Han Kim, https://orcid.org/0000-0003-4868-9145

\section{REFERENCES}

1. Min BG. [Diagnosis and treatment of hair \& scalp disorders]. Seoul: Hanmi Book; 2005. p. 21-30. Korean.

2. Kim GO, Kim GO, Kim HK, Kim HR, Im EJ, Kim TY, Jung KS, et al. Scalp hair care for trichologists. Seoul: Chungram Publisher; 2010. p. 52-60.

3. Healthcare Bigdata Hub [Internet]. Wonju: Health Insurance Review \& Assessment Service; c2021 [cited 2021 Jun 24]. Available from: http://opendata.hira.or.kr/op/opc/olapMfrnIntrsIlnsInfo.do.

4. So HR, We SY, Im EJ. Comparison of hair loss factors by sex in Korean adult. J Korean Soc Cosmetol. 2011;17(2):286-96.

5. Jung JY, Han KH. Common skin diseases in Korea. Seoul: MD World; 2009. p. 495-522.

6. Hanneken S, Ritzmann S, Nöthen MM, Kruse R. [Androgenetic alopecia. Current aspects of a common phenotype]. Hautarzt. 2003;54(8):703-12. German.

7. Alkhalifah A. Alopecia areata update. Dermatol Clin. 2013; 31(1):93-108

8. Libecco JF, Bergfeld WF. Finasteride in the treatment of alopecia. Expert Opin Pharmacother. 2004;5(4):933-40.

9. Kim DY, Park SJ. Pharmacological therapeutics in androgenetic alopecia. J Korean Med Assoc. 2020;63(5):277-85.
10. Pinnell SR, Murad S. Effects of minoxidil on cultured human skin fibroblasts. Dermatologica. 1987;175 Suppl 2:12-8.

11. Ha WH, Park DH. Effect of seaweed extract on hair growth promotion in experimental study of C57BL/6 mice. Arch Craniofac Surg. 2013;14(1):1-10.

12. Jang HY, Choi KH, Kim SH, Kwon KR, Kim BW. Bibliographic studies of depilation. J Pharmacopuncture. 2002;5(2):92-108.

13. Kim YJ, Moon JB, Yi TH. The study of literature on meridians and acupoints about acupuncture treatment of alopecia. J Korean Orient Med. 2006;27(3):212-21.

14. Choi SB, Kang JA, Lee JH. Five cases of severe alopecia areata treated with Gagamhwajung-hwan. J Korean Med Ophthalmol Otolaryngol Dermatol. 2017;30(3):239-49.

15. Ha KS, Song IS, Lee JL, Na HS, Shin JN. A case report of a child in alopecia totalis. J Korean Orient Pediatr. 2004;18(2):191-200.

16. Lee SW, Ko JM, Lee SY, Lee MH, Kim YJ, Lee SH, et al. A case study of beevenom effect on Alopecia Universalis started from alopecia areata. J Korean Acupunct Moxib Soc. 2008;25(6):16373.

17. Kim CY, Seo HS, Lee DJ, Kwon K. Case study on treating scarring alopecia and alopecia arreata using low level laser therapy and acupuncture. J Korean Med Ophthalmol Otolaryngol Dermatol. 2016;29(4):182-8.

18. Ju BH, Choi YK, Yu SA, Lee SY. A case report on child with different types of alopecia. J Pediatr Korean Med. 2013;27(3):53-64.

19. Yoon HJ. A case study of androgenetic alopecia in woman improved by pharmacopuncture therapy and needle-embedding therapy. J Korean Med Ophthalmol Otolaryngol Dermatol. 2014;27(3):162-70.

20. Choi JW, Lee JH, Jung BY, Ji YJ, Lee KS. Retrospective study about the effectiveness of Korean medical treatment on 222 patients with alopecia areata. J Korean Med Ophthalmol Otolaryngol Dermatol. 2019;32(4):13-28.

21. Yun JH, Kim KH, Jang SJ, Sin MS. One case treated alopecia areata with herbal acupuncture. J Orient Med Surg Ophthalmol Otolaryngol. 2001;14(1):105-10.

22. Kim KW, Seo JC, Lee KM, Lim SC, Jung TY, Han SW. A case of alopecia areata treated with bee venom and carthami flos herbal acupuncture. J Pharmacopuncture. 2004;7(2):91-6.

23. Lee IJ, Kim HJ. The case study of Korean medicine on patients with alopecia areata accompanied by atrophodermia. Semyung Orient Med Inst. 2009;12:39-52.

24. Hwang BM, Jeong MJ, Seo HS. A case report of child with alopecia areata. J Korean Orient Pediatr. 2012;26(2):47-52.

25. Ko SJ, Park JW. A case study of male pattern alopecia in man improved by $\mathrm{V}$ and HA Yakchim and Korean medicine treatments. J Korea Immuno-Yakchim Soc. 2016;5(1):47-55.

26. Kim JS, Park SY. A case of alopecia areata treated with Korean 
medical treatment. Herb Formula Sci. 2016;24(2):124-30.

27. Yoon YJ, Kwon NH, Shin HJ, Jang JY. Four cases of androgenetic alopecia patient using Korean medicine treatment and Western treatment. J Korean Med Ophthalmol Otolaryngol Dermatol. 2017;30(2):170-7.

28. Choi HS, Lee SJ, Kim BNR. A case report of the retreatment with the same herbal medicine after the treatment of alopecia areata in a child. J Pediatr Korean Med. 2019;33(4):89-99.

29. Zhang YM, Liu CH, Wang YC, Teng HL, Meng XL, Han XJ. Medicated thread moxibustion for alopecia areata: a case report. Medicine (Baltimore). 2019;98(44):e17793.

30. Yun GW, Jeong SH, Shin KS. Two cases of androgenetic alopecia patients treated with traditional oriental hair care products and Yukmijihwang-huan. J Korean Med Ophthalmol Otolaryngol Dermatol. 2020;33(1):75-82.

31. Kim MY, An BJ. A study of hair loss prevention and hair growth promotion by Korean traditional fomular. Korea J Herbol. 2004; 19(2):9-20.

32. Yi TH, Moon JB, Kim YJ, An KE. Oriental medicine therapy in the treatment of men with androgenetic alopecia. J Korean Acupunct Moxib Soc. 2006;23(1):179-86.
33. Lee JH, Jang JY, Yoon YJ, Cho AR, Shin HJ, Jung SU, et al. Retrospective study about the causes and the effects of Korean medicine treatment on alopecia- focused on acquired febrile alopecia. J Korean Med Ophthalmol Otolaryngol Dermatol. 2013;26(2): 30-44.

34. Hong JA, Kang SJ, Jang JY, Kang YR. A clinical trial on efficacy of Gagamchengyoung-tang(Jiājiănqinngyíng-tāng) in the alopecia patients with febrile tendency. J Korean Med Ophthalmol Otolaryngol Dermatol. 2013;26(2):19-29.

35. Starace M, Alessandrini A, Brandi N, Piraccini BM. Preliminary results of the use of scalp microneedling in different types of alopecia. J Cosmet Dermatol. 2020;19(3):646-50.

36. Wu T. Wenbingtiaobian. Seoul: Jipmoondang; 2004. 738 p.

37. Park YY. Medicine wire Injection therapy. Seoul: Haeng Lim Seo Wen; 2003. p. 19, 23.

38. Han JS, Lee KR. A research of the effectiveness of far infrared ray and TDP therapy on the hair loss treatment. Asian J Beauty Cosmetol. 2010;8(1):187-99.

39. Kweon SH, Hong SH. The current status of Korean medicine treatment of alopecia: focusing on case reports. J Korean Med Ophthalmol Otolaryngol Dermatol. 2019;32(3):164-85. 